\title{
Clinical spectrum and outcome of invasive mucormycosis in children and adults: Polish experience of the decade 2010-2019
}

\begin{abstract}
No epidemiological data exist so far on invasive mucormycosis (IM) in Polish hematopoietic cell transplantation (HCT) and pediatric hemato-oncology (PHO) centers. The objective of this study was to analyze the incidence, clinical course, therapy, and outcome of IM in pediatric and adult patients undergoing HCT and children with hemato-oncological diseases in Poland. A total number of 12425 at-risk patients were retrospectively analyzed, and the period between 2010 and 2019 was included. Patients were analyzed in three groups: nontransplant children with malignancies, children undergoing HCT, and adults after HCT. Twenty-one patients were diagnosed with IM, including 15 children (10 non-HCT, 5 HCT) and 6 HCT adults. Proven IM was confirmed in 18 patients, probable in 2 patients, and possible in 1 patient. Proven IM was diagnosed in $7.1 \%$ of all patients with invasive fungal diseases (IFDs), including 8.1\% among PHO patients, 5.4\% among pediatric HCT patients, and 7.0\% among adult HCT patients. Clinically, pneumonia was diagnosed in $10(47.6 \%)$ patients, involvement of the paranasal sinuses was found in $3(14.3 \%)$ patients, gastrointestinal disease was noted in $2(9.5 \%)$ patients, and disseminated mucormycosis was found in $6(28.6 \%)$ patients. The probability of overall survival in IM patients was $0.50 \pm 0.11$. Infection-related mortality (IRM) was $10 \%$ for pediatric nontransplant IM patients and $82 \%$ for transplant IM (pediatric + adult) patients $(p=0.004)$. Among the transplant patients, all adults died within 120 days. IRM for pediatric HCT patients was $60 \%$ $(p=0.038)$. The only prognostic factor was HCT, which adversely influenced survival in IM patients.
\end{abstract}

(๑) 2020 Polish Society of Hematology and Transfusion Medicine, Insitute of Hematology and Transfusion Medicine. Published by Sciendo. All rights reserved.

Keywords:

invasive fungal disease, invasive mucormycosis, children, adults, leukemia, hematopoietic cell transplantation

\section{Introduction}

Invasive mucormycosis (IM) is responsible for $8 \%$ of invasive fungal diseases (IFDs) in patients with hematological malignancies (HMs) or after hematopoietic cell transplantation (HCT), and its incidence is significantly increasing in these cohorts. These two groups of patients, together with diabetes patients, are regarded to be at the highest risk of development of IM [1, 2, 3].

Mucormycetes (previously: Zygomycetes; recently proposed: Entomophthorales [4]) organisms are highly aggressive, filamentous, ubiquitous, fast-growing, broad aseptate fungi, which are unique among filamentous fungi because of their disproportionately high capacity to cause devastating disease in patients regardless of underlying conditions $[5,6]$.

The activity of Mucormycetes is characterized by angioinvasion and tissue invasion, which leads to a rapid and progressive infection. The clinical presentations are pulmonary, gastrointestinal, soft tissue, paranasal (sino-orbital), and cerebral mucormycoses, with possible evolution into disseminated disease $[6,7]$.
No epidemiological data exist so far about IM in Polish $\mathrm{HM} / \mathrm{HCT}$ centers. The aim of this paper was to present the analysis of incidence, clinical course, therapy, and outcome of IM in children and adults either with hematooncological diseases or undergoing HCT in Poland.

\section{Patients and methods}

\section{Design of the study}

Collection and analysis of data were performed as part of the project on analysis of "Infections in pediatric hematology, oncology, and hematopoietic transplantation" ("iPhot") in the respective 2-yearperiod editions of 2013, 2015, 2017, and 2019; this study was devoted to the analysis of the incidence and outcome of fungal, bacterial, and viral infections in $\mathrm{HCT} /$ pediatric hemato-oncology $(\mathrm{PHO})$ patients. This project was widened to include the adult hematopoietic transplant setting for the period 20122014. The overall results of this national project were presented elsewhere [8, 9, 10].

Additionally, published literature and abstracts from national hematology meetings (Polish Society of
Article history:

Received: 02.02.2020

Accepted: 03.03.2020

Jan Styczyński ${ }^{*}$

Krzysztof Czyżewski ${ }^{1}$, Jowita Frączkiewicz²,

Małgorzata Salamonowicz ${ }^{2}$,

Agnieszka Piekarska, ${ }^{1,3}$,

Monika Adamska,

Przemysław Gałązka ${ }^{1,5}$

Patrycja Mensah-Glanowska ${ }^{6}$

Joanna Drozd-Sokołowska ${ }^{7}$,

Anna Waszczuk-Gajda 7

Agnieszka Tomaszewska, ${ }^{7,15}$

Kazimierz Hałaburda ${ }^{15}$

Marcin Płonowski ${ }^{8}$,

Olga Gryniewicz-Kwiatkowska ${ }^{9}$,

Patrycja Zalas-Więcek ${ }^{10}$

Liliana Chełmecka-Wiktorczyk ${ }^{11}$,

Ninela Irga-Jaworska ${ }^{12}$,

Tomasz Ociepa ${ }^{13}$

Renata Tomaszewska ${ }^{14}$,

Lidia Gil ${ }^{4}$ for Polish Society

of Pediatric Oncology and

Hematology and Polish Adult

Leukemia Group

Department of Pediatric Hematology an

Oncology, Collegium Medicum, Nicolaus

Copernicus University Toruń, Bydgoszcz, Poland

'Department of Pediatric Transplantology

Hematology and Oncology, Medical University,

Wroctaw, Poland

Department of Hematology, Medical University, Gdańsk, Poland

Department of Hematology, Poznań University of Medical Sciences, Poznań, Poland

Department of Pediatric Surgery, Collegium

Medicum, Nicolaus Copernicus University Toruń,

Bydgoszcz, Poland

Department of Hematology, Collegium Medicum Jagiellonian University, Kraków, Poland; 'Department of Hematology, Medical University, Warszawa Poland

Warszawa, Poland

Department of Pediatric Oncology and

Hematology, Medical University, Biatystok, Poland

'Department of Oncology, Children's Memorial

Health Institute, Warszawa, Poland

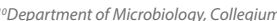

Dedicum Nicolaus Copernicus University Toruhi

Bydgoszcz, Poland

"Department of Pediatric Oncology and Hematology, University Children's Hospital Jagiellonian University Collegium Medicum Kraków, Poland

.Departand Department of Pediatrics, Hematology and Oncology, Medical University, Gdonsk, Poland ${ }^{3}$ Department of Pediatric Hemato-Oncology and Gastroenterology, Pomeranian Medical University, Szczecin, Poland

"Department of Pediatric Hematology and Oncology Sitesian Medical University Zabree Poland

${ }^{15}$ Department of Hematology, Institute of Hematology and Transfusion Medicine, Warszawa Poland

${ }^{*}$ Corresponding author: Jan Styczyński, Department of Pediatric Hematology and Oncology, Collegium Medicum, Nicolaus Copernicus University Toruń, Skłodowskiej-Curie 9, 85-094 Bydgoszcz, Poland, phone: +4852 5854860, fax: +4852 5854087, email: jstyczynski@cm.umk.pl 
Pediatric Oncology and Hematology [PTOHD], Polish Society of Hematology and Transfusion Medicine [PTHiT] and Polish Adult Leukemia Group [PALG]) during the period 2010-2012 were retrieved and searched for data on infections with mucormycoses in hematopoietic transplant, hematology, and $\mathrm{PHO}$ settings in Polish hematology or HCT settings. Nevertheless, no data on mucormycoses for the period 2010-2011 were found in available literature.

\section{Patients}

Three groups of patients were included in the study. Group A: all consecutive adult patients transplanted (Ad-HCT) between January 1, 2012 and December 31, 2014 in 11/13 Polish HCT centers for adults, as well as patients after HCT diagnosed with mucormycosis between January 1, 2015 and September 30, 2019 in 7 HCT centers for adults. Group B: all consecutive pediatric patients transplanted (Ch-HCT) between January 1, 2012 and December 31, 2017 in 5/5 pediatric HCT centers, and post-HCT patients diagnosed with mucormycosis between January 1, 2018 and July 31, 2019 in pediatric HCT centers. Group C: all consecutive children treated between January 1, 2012 and December 31, 2017 for malignant hematological and nonhematological diseases in 14-16 out of 18 pediatric oncology centers, as well as children with malignancies diagnosed with mucormycosis between January 1, 2018 and July 31, 2019 in PHO centers. Infections were reported every 2 years by each center, and data were verified and analyzed centrally by three researchers.

\section{Diagnosis of fungal infections}

The diagnosis of IFD was made according to the criteria of the European Organization for the Research and Treatment of Cancer/ Mycosis Study Group (EORTC/MSG) as proven, probable, or possible in the 2002 and 2008 versions, and finally revised and updated according to the new 2019 criteria [11]. All HCT and PHO patients were screened with the galactomannan test mainly during neutropenia or on the basis of clinically driven indications $[8,9,10]$. Mucormycetes-directed polymerase chain reaction (PCR) study was not used in the diagnosis for the patients included in this analysis.

\section{Definitions of proven, probable, and possible mucormy- cosis}

Due to the lack of standardized biomarkers specific for Mucormycetes during the study period, the definitions of proven, probable, and possible IM were modified for the purpose of this study.

Proven IM diagnosis required histopathologic, cytopathologic, or direct microscopic examination or positive culture of a specimen obtained by biopsy, from the site corresponding to clinical symptoms. Probable IM diagnosis required the presence of clinical features and (i) mycologic evidence or (ii) histopathologic, cytopathologic, or direct microscopic examination or positive culture of a specimen obtained by biopsy, from the site NOT corresponding to clinical symptoms (e.g., clinical and radiological symptoms of invasive fungal pneumonia and presence of Mucormycetes in the gastrointestinal tract; with the absence of evidences of any other documented infectious etiology).
Possible IM was diagnosed in cases that met the criteria for a host factor and a clinical feature but for which mycological evidence was not found.

\section{Prophylaxis of fungal infections}

Environmental prophylaxis was applied in all the centers according to the commonly accepted policy $[12,13]$. Children received antifungal prophylaxis with fluconazole till 2014. Afterward, posaconazole and subsequently voriconazole were introduced in the national program of antifungal prophylaxis in children; therefore, from the end of 2014, posaconazole or voriconazole were used in high-risk acute leukemia patients, in the neutropenic phase after $\mathrm{HCT}$, in the case of graftversus-host-disease (GVHD), or in secondary prophylaxis. In the adult HCT setting, fluconazole was used for antifungal prophylaxis during neutropenia, together with regular screening using serum galactomannan level and high-resolution computed tomography (HRCT/CT) in case of suspected IFD; from 2015 onward, posaconazole or voriconazole were used in case of GVHD.

\section{Data analysis}

Clinical characteristics and outcome were performed on all cases of mucormycoses reported by the participating centers between 2010 and 2019. The overall incidence of IM for adults with respect to the number of patients treated for malignancy or undergoing transplantation was calculated based on the following data: from 2012 to 2014 from 11 centers; from 2015 to 2019 from 4 centers. Similarly, for children, this was calculated based on data from 2012 to 2017 from 14-16 centers and data from 2018 to 2019 from 3 centers. The incidence of IM as a rate of all cases of IFD for adults, with respect to the number of patients with IFD, was calculated based on data from 2012 to 2014 from 11 centers; while for children, this was done based on data from 2012 to 2017 from 14-16 centers.

\section{Infection-related mortality (IRM)}

Treatment-related mortality was defined as any death occurring at any point after the start of treatment, which did not occur as a result of relapse or secondary malignancy. Outcome of infection was regarded as positive in case of survival in infected people or negative in case of death from infection. IRM was defined as any death that occurred in the presence of infection, starting from the day of diagnosis of infection. In case of relapse and progression of malignancy, this was regarded as the primary cause of death, regardless of concomitant infection.

\section{Transplant procedures and unrelated donor matching}

For unrelated donors, human leukocyte antigen (HLA) compatibility was established for 10 antigens. For matched sibling donors (MSDs), HLA compatibility was established for 6 antigens. In case of unrelated donors, 10/10 HLA compatibility was regarded as matched unrelated donor (MUD), while <10/10 HLA was regarded as mismatched unrelated donor (MMUD). Most patients who underwent MUD/ MMUD-HCT received antithymocyte globulin (ATG). 


\section{Statistical analysis}

Categorical variables were compared using the chi-square test, noncategorical variables were compared using the Mann-Whitney $U$-test. Odds ratios (ORs) and confidence intervals (95\% Cls) were calculated for the difference in the occurrence of infections in the patients. For the analysis of incidence, an infectious event was defined as the diagnosis of a first specific infectious disorder. Cumulative incidence of infections was calculated, starting from the day of transplant in the HCT setting (or the day of diagnosis of malignancy in the PHO setting) to the day of the first infection. Death was considered the competing event. The Kaplan-Meier method was used to determine survival after infection, counting from the day of diagnosis of infection. The relationship between death from mucormycosis and other variables regarded as risk factors was examined using univariate logistic regression: ORs and $95 \% \mathrm{Cls}$ were calculated for each factor. All reported $p$-values are two-sided; $p<0.05$ was considered statistically significant.

\section{Results}

\section{Demographics}

A total number of 21 patients were diagnosed with IM, including $15 / 7788(0.019 \%)$ children and $6 / 5237(0.011 \%)$ adults $(O R=2.9$;
95\% Cl $=2.0-3.6 ; p<0.0001$, Tab. I). There were no differences in the incidence of mucormycosis between $\mathrm{PHO}$ and HCT (pediatric + adults) patients $(0.017 \%$ vs. $0.017 \%$; OR $=1.0$; $95 \% \mathrm{Cl}=0.4-2.2 ; p=0.9)$. The incidence of mucormycosis was much higher in pediatric HCT than adult HCT patients (0.043\% vs. $0.011 \%$; OR $=3.8 ; 95 \% \mathrm{Cl}=1.1-12.3 ; p=0.019)$. Out of 21 patients with mucormycosis, proven infection was confirmed in 18 patients, probable in 2 patients, and possible in 1 patient.

\section{Relative IM incidence}

The analysis of relative incidence of IM among all diagnosed IFD cases was possible for $495 \mathrm{PHO}, 206$ pediatric $\mathrm{HCT}$, and 183 adult HCT patients, who were diagnosed with IFD at different levels (proven, probable, or possible) (Tab. II). When only proven diagnosis of IFD was taken into account, IM was diagnosed in $7.1 \%$ of all patients, with specific incidence of $8.1 \%$ among $\mathrm{PHO}$ patients, $5.4 \%$ among pediatric HCT patients, and $7.0 \%$ among adult HCT patients ( $p=n s ;$ for all comparisons).

\section{Etiology of mucormycosis}

Histopathologic or microbiological diagnosis showed the presence of Mucor spp. in 13 patients (including 2 with probable clinical level), Rhizopus spp. in 4 patients (including $R$. mucoralis in 2 and $R$. oryzae

Table I. Epidemiology of mucormycosis among all patients at risk

\begin{tabular}{|l|c|c|c|c|}
\hline Groups of patients & Children: PHO & Children: HCT* & Adults: HCT & Total \\
\hline Total & $10 / 6024$ & $5 / 1164$ & $6 / 5237$ & $21 / 12425$ \\
\hline Allo-HCT & - & $5 / 889$ & $6 / 2037$ & $11 / 2926$ \\
\hline Auto-HCT & - & $0 / 275$ & $0 / 3200$ & $0 / 3475$ \\
\hline ALL & $8 / 1317$ & $4 / 293$ & $0 / 348$ & $12 / 1958$ \\
\hline AML & $0 / 295$ & $1 / 171$ & $3 / 1008$ & $4 / 1474$ \\
\hline NHL & $1 / 352$ & $0 / 45$ & $0 / 756$ & $1 / 1153$ \\
\hline HL & $0 / 371$ & $0 / 20$ & $1 / 440$ & $1 / 831$ \\
\hline CML/MPD & $0 / 37$ & $0 / 12$ & $1 / 181$ & $1 / 230$ \\
\hline Other & $1(\mathrm{SHML}) / 109(\mathrm{LCH})$ & - & $1(\mathrm{CLL})$ & - \\
\hline
\end{tabular}

* - an overlap of pediatric HCT patients with PHO patients is possible; however, PHO patients were censored at the moment of HCT; ALL - acute lymphoblastic leukemia; AML - acute myeloid leukemia; NHL - non-Hodgkin lymphoma; HL - Hodgkin lymphoma; CML - chronic myelogenous leukemia; MPD - myeloproliferative disease; LCH - Langerhans cell histiocytosis; SHML - Sinus histiocytosis with massive lymphadenopathy; CLL - chronic lymphocytic leukemia; HCT - hematopoietic cell transplantation; PHO - pediatric hemato-oncology

Table II. Epidemiology of mucormycosis among patients diagnosed with invasive fungal disease

\begin{tabular}{|l|c|c|c|c|}
\hline \multicolumn{1}{|c|}{ Groups of patients } & Children: PHO & Children: HCT & Adults: HCT & Total \\
\hline Total & $8 / 495(1.6 \%)$ & $2 / 206(1.0 \%)$ & $3 / 183(1.6 \%)$ & $5 / 884(1.5 \%)$ \\
\hline Allo-HCT & - & $2 / 197(2.0 \%)$ & $3 / 132(2.3 \%)$ & $0 / 75(0.0 \%)$ \\
\hline Auto-HCT & - & $0 / 24(0.0 \%)$ & $0 / 51(0.0 \%)$ & $7 / 308(2.3 \%)$ \\
\hline ALL & $6 / 226(2.7 \%)$ & $1 / 58(1.7 \%)$ & $0 / 24(0.0 \%)$ & $3 / 208(1.4 \%)$ \\
\hline AML & $0 / 84(0.0 \%)$ & $1 / 51(2.0 \%)$ & $2 / 73(2.7 \%)$ & $1 / 51(2.0 \%)$ \\
\hline NHL & $1 / 33(3.0 \%)$ & $0 / 4(0.0 \%)$ & $0 / 14(0.0 \%)$ & - \\
\hline Other & $1(\mathrm{SHML})$ & - & $1(\mathrm{CLL})$ & \\
\hline Level of diagnosis & \multicolumn{5}{|c|}{} \\
\hline Proven & $6 / 74(8.1 \%)$ & $2 / 37(5.4 \%)$ & $3 / 43(7.0 \%)$ & $11 / 154(7.1 \%)$ \\
\hline Probable & $2 / 98(2.0 \%)$ & $0 / 55(0.0 \%)$ & $0 / 49(0.0 \%)$ & $2 / 202(1.0 \%)$ \\
\hline Proven + probable & $8 / 172(4.6 \%)$ & $2 / 92(2.2 \%)$ & $3 / 92(3.3 \%)$ & $11 / 356(3.1 \%)$ \\
\hline
\end{tabular}

* - an overlap of pediatric HCT patients with PHO patients is possible; however, PHO patients were censored at the moment of HCT; ALL - acute lymphoblastic leukemia; AML - acute myeloid leukemia; NHL - non-Hodgkin lymphoma; HL - Hodgkin lymphoma; CML - chronic myelogenous leukemia; MPD - myeloproliferative disease; LCH - Langerhans cell histiocytosis; SHML - Sinus histiocytosis with massive lymphadenopathy; CLL - chronic lymphocytic leukemia 
in 1 patient), Absidia spp. (Lichtheimia corymbifera) in 2 patients, and Cunninghamella spp. in 1 patient (Tab. III). In 8/21 (38.1\%) cases, coexisting IFD was diagnosed: invasive aspergillosis in 4 patients ( 3 A. fumigatus and 1 A. spp.), and invasive candidiasis in 4 patients (Candida albicans; C. glabrata + C. albicans; C. glabrata + C. krusei; C. parapsilosis).

\section{Table III. Etiology of mucormycosis}

\section{\begin{tabular}{l|l|l|} 
Children: PHO $(n=10)$ & Children: HCT $(n=4+1)$ & Adults: HCT $(n=6)$
\end{tabular}}

\begin{tabular}{l|l|l} 
Mucor spp.: 8 & Rhizopus mucoralis: 2 & Mucor spp.: 4 \\
Cunninghamella spp.: 1 & Mucor spp.: 1 & Rhizopus oryzae: 1 \\
Absidia spp.: 1 & Absidia spp.: 1 & Rhizomucor spp.: 1 \\
& NA (possible IM): 1 &
\end{tabular}

PHO - pediatric hemato-oncology; HCT - hematopoietic cell transplantation; IM - invasive mucormycosis

\section{Clinical course}

Risk factors for the development of IM included neutropenia in 14 patients and steroid therapy in 7 patients. Additionally, 4 patients were on other immunosuppressive therapy, 2 patients had steroiddependent diabetes, and 1 patient was concomitantly treated for CMV infection. On diagnosis of mucormycosis, 13/21 patients were on antifungal prophylaxis, including fluconazole in 7 patients and posaconazole in 6 patients - in a few cases, given sequentially with fluconazole $(n=2)$, voriconazole $(n=1)$, or micafungin $(n=1)$. Time to the development of IM from the beginning of chemotherapy in the $\mathrm{PHO}$ setting or from the day of transplantation in the HCT setting was comparable (Fig. 1).

A
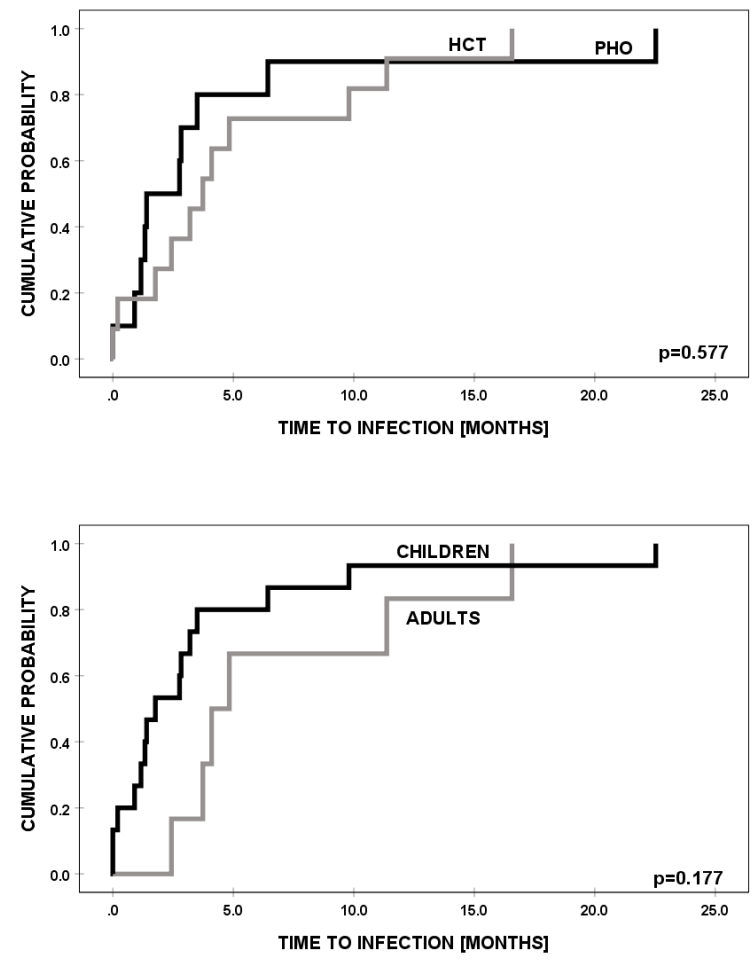

Fig.1. Time to development of IM: (A) PHO vs. HCT patients, (B) pediatric vs. adult patients
Clinically, pneumonia was diagnosed in 10 (47.6\%) patients, paranasal sinus involvement in $3(14.3 \%)$ patients, gastrointestinal disease in $2(9.5 \%)$ patients, and disseminated mucormycosis in 6 $(28.6 \%)$ patients.

\section{Therapy and survival of mucormycosis}

In 10 patients, antifungal monotherapy was administered, including sequential use of antifungals. Liposomal amphotericin $B(L A m B)$ was used in 6 patients, posaconazole in 5 patients, and voriconazole in 1 patient (postmortem diagnosis of IM). Combination secondline antifungal therapy was given in 11 patients: it was based on amphotericin B lipid complex (ABLC) in 7 cases and based on LAmB in 4 patients. Data on surgical treatment were available for 4 patients. Overall, 11/21 (52.4\%) patients had survived IM. Kaplan-Meier-based probability of survival in IM patients was $0.503 \pm 0.112$ (Fig. 2). IM IRM was $10 \%$ for pediatric nontransplant (PHO) patients and $82 \%$ for transplant (pediatric + adult) patients $(p=0.004)$. Among transplant patients, all adults died within 120 days, and IRM for pediatric HCT patients was $60 \%(p=0.038)$ (Fig. 3).

Details on successful antifungal therapy (only children: $9 \mathrm{PHO}$ and 2 HCT patients) are presented in table IV. Overall, combination therapy was successful only in 3/11 patients, indicating the severity of the disease or failed first-line therapy.

\section{Risk factor analysis of survival in IM patients}

In univariate logistic regression of risk factor analysis of survival in IM patients, the following factors were analyzed: children vs. adults, transplant vs. nontransplant setting, clinical form, coexisting fungal infection, combination therapy used, use of any form of amphotericin, and use of LAmB vs. ABLC.

Although nonsignificant, a trend toward better outcome was observed in the case of sinus or gut involvement, as well as use of LAmB. No analysis could be performed for age groups, as all adults with IM had died. The only prognostic factor was HCT setting, which adversely influenced survival in IM patients $(\mathrm{OR}=40 ; 95 \% \mathrm{Cl}=3.5-530$; $p=0.005)$.

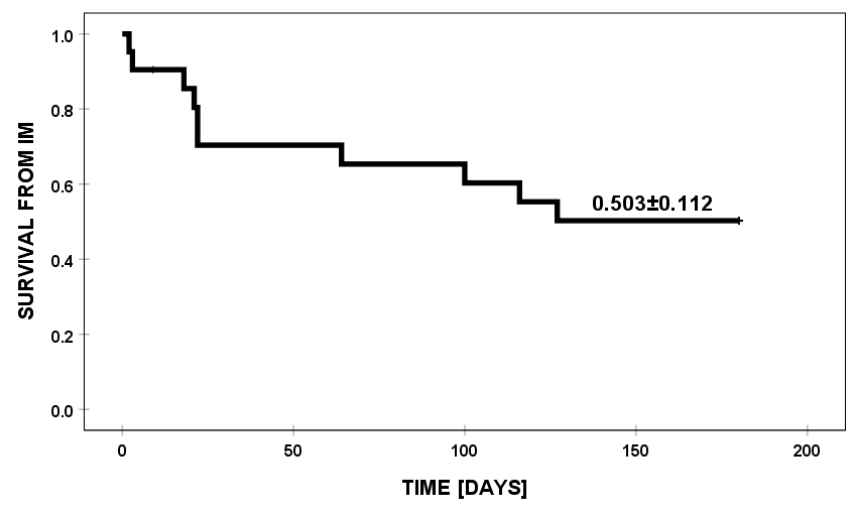

Fig. 2. Survival in 21 patients with invasive mucormycosis 


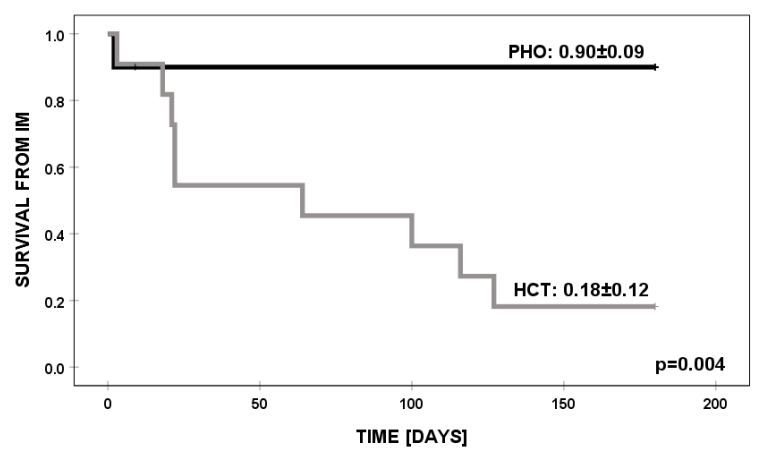

C

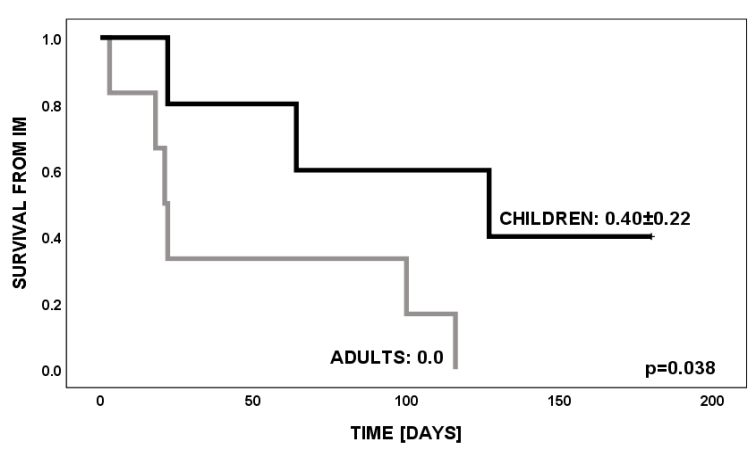

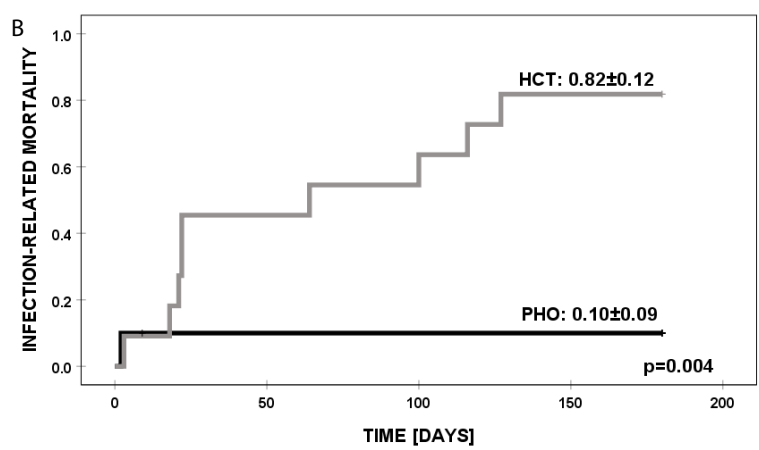

D

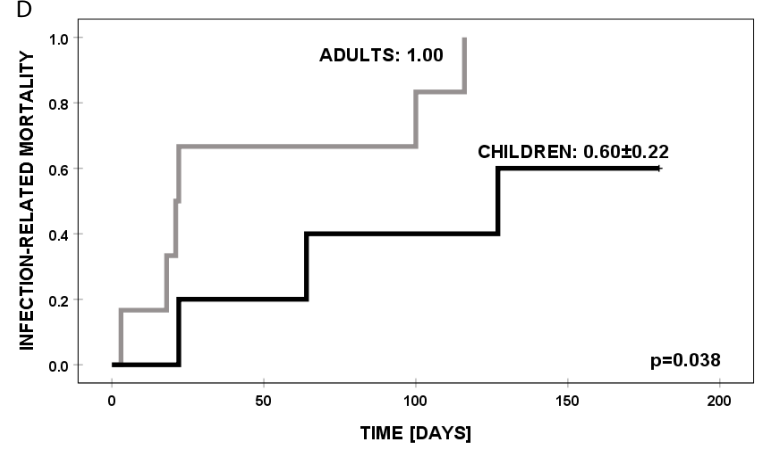

Fig. 3. Survival in IM patients and infection-related mortality in mucormycosis: $(A, B)$ in PHO vs. HCT (children + adult) patients, (C, D) in HCT children vs. adult HCT patients

\section{Table IV. Successful antifungal therapy}

\begin{tabular}{|c|c|c|}
\hline Patient & Clinical form & Antifungal therapy \\
\hline 1 & Paranasal sinuses^ & LamB + Posaconazole* \\
\hline 2 & Paranasal sinuses^ & LAmB, Posaconazole, ABLC, Posaconazole \\
\hline 3 & Pulmonary & Posaconazole \\
\hline 4 & Pulmonary & Voriconazole, Caspofungin + ABLC*, Isavuconazole \\
\hline 5 & Disseminated & Voriconazole, Posaconazole, LAmB \\
\hline 6 & Gastrointestinal & $\mathrm{LAmB}$ \\
\hline 7 & Pulmonary & $\mathrm{LAmB}$ \\
\hline 8 & Pulmonary & Voriconazole $+\mathrm{ABLC}^{*}$, Posaconazole \\
\hline 9 & Pulmonary & Voriconazole, LAmB \\
\hline 10 & Gastrointestinal & Posaconazole \\
\hline 11 & Paranasal sinuses & LAmB \\
\hline
\end{tabular}

* - combination therapy, ^ - after HCT; LAmB- liposomal amphotericinB; ABLC - amphotericin B lipid complex

\section{Discussion}

In this study, we analyzed epidemiology, treatment, and outcome of IM in 12425 patients at risk. Most of the Polish HCT and pediatric oncology centers participated in the study, with period of inclusion ranging from 3 to 6 years, rendering the results of important value. We have shown that proven IM was diagnosed in $7.1 \%$ of all patients with IFD, as well as in specific subgroups: $8.1 \%$ among $\mathrm{PHO}$ patients, $5.4 \%$ among pediatric HCT patients, and $7.0 \%$ among adult HCT patients. These results are comparable with other reports [1, 14];
Leventakos et al. [15] even indicated an increase in relative incidence of IM up to $9 \%$ in an autopsy study.

In our material, Mucor spp. was the most frequently (70\%) diagnosed species in hematological patients, while in European material, it was Rhizopus spp. (34\%). Absidia and Cunninghamella were relatively rare in both our sample and the European material [16]. Similar to other data, the most frequent clinical type was pulmonary or disseminated disease.

Probability of overall survival in IM patients was $0.50 \pm 0.11$. This is a much better result than the results from studies performed a decade 
ago $[5,16,17]$ and is comparable with recent reports $[6,7,18]$. Still, the outcome is much worse than in immunosuppressed patients infected with invasive aspergillosis or invasive candidiasis. However, it should be underlined that the outcome for pediatric patients, both transplant and nontransplant, was much better, reaching $90 \%$ in nontransplant patients. Good outcome was seen in cases of gut or paranasal sinus involvement, although the number of patients with such presentation was small.

Introduction of the national program of antifungal prophylaxis with posaconazole in 2014-2015 has already resulted in a decrease of IFD cases for the following groups of patients - patients with high-risk acute leukemia, HCT patients with neutropenia and GVHD, and patients in secondary prophylaxis. On the other hand, voriconazole, the other antifungal agent frequently used in prophylaxis, could influence the rate of IM, since the spectrum of activity of this azole does not cover Mucorales.

In the majority of patients with positive outcome of IM, treatment was based on the use of LAmB, although other drugs or combinations were also successful in individual cases (Tab. IV). Recent data indicate that isavuconazole is another important antifungal agent with activity against Mucorales [18, 19, 20]. Several recent meta-analyses have also addressed the topic of mucormycosis [21-24].

The limitations of the study include the following: different periods of inclusion of specific centers, limited possibility of diagnosis of IM with PCR in some of the centers, and limited number of biopsies performed in case of suspicion of IFD. We are aware that the incidence of IM could possibly be underdiagnosed, as there are no biomarkers available and Mucor-directed PCR has become available only recently in Poland. The diagnosis of IM in clinical practice is based on biopsy only, as this invasive procedure is not frequently performed in HCT patients and in children with HMs.

\section{Conclusions}

Current data from Polish pediatric and adult HCT centers as well as pediatric oncology centers, show the incidence of IM at the rate of $7.1 \%$ of all proven cases of IFD. The most frequent species are Mucor spp. and Rhizopus spp. Clinically, pulmonary or disseminated mucormycosis presented in $75 \%$ of all cases. Although no statistical analysis could be performed, most of the IM cases occurred among allo-HCT, pediatric acute lymphoblastic leukemia, and adult acute myeloid leukemia patients. The outcome of IM is dismal, particularly in patients after allo-HCT; however, treatment of IM in nontransplant children resulted in $90 \%$ success. The use of LAmB - and in individual cases, posaconazole, ABLC, or isavuconazole - contributed to positive outcome and survival in IM patients. Finally, the incidence and outcome of IM in Polish post-HCT patients and in Polish children treated for malignancy are similar to European data.

\section{Acknowledgments}

The authors would like to thank the heads and physicians of the hematopoietic cell transplant centers and departments of pediatric oncology and hematology: Jerzy Kowalczyk, Mariusz Wysocki, Tomasz Szczepański, Michał Matysiak, Jacek Wachowiak, Bożenna Dembowska-Bagińska, Walentyna Balwierz, Elżbieta Drożyńska, Tomasz Urasiński, Jarosław Peregud-Pogorzelski, Maryna Krawczuk-Rybak, Wojciech Młynarski, Krzysztof Kałwak, Radosław Chaber, Mariola Woszczyk, Wanda Badowska, Grażyna Karolczyk, Grażyna Sobol-Milejska, Agnieszka Mizia-Malarz, Sebastian Giebel, Sławomira Kyrcz-Krzemień, Jolanta Goździk, Grzegorz W Basak, Krzysztof Kałwak, Marek Hus, Tadeusz Robak, Agnieszka Wierzbowska, Piotr Rzepecki, Jan Maciej Zaucha, Tomasz Wróbel, Beata Piątkowska-Jakubas, Olga Zając-Spychała, Agnieszka Zaucha-Prażmo, Jarosław Dybko, Joanna Mańko, Alicja SadowskaKlasa, Monika Biernat, Aneta Czajńska-Deptuła, Magdalena Bartnik, Paweł Wawryków, Ewa Bień, Zofia Małas, Agnieszka UrbanekDądela, Filip Pierlejewski, Anna Szmydki-Baran, Zuzanna GamrotPyka, Weronika Stolpa, and Jakub Musiał for their support from 2012 for the project on infectious diseases and/or for provision of reference nonmucormycosis data, published elsewhere.

\section{Authors' contributions}

JS, LG - study design, manuscript writing, and administrative support. JS, KC, PG - data analysis and interpretation, data checkup, and statistical analysis. All authors - provision of important clinical data and give their final approval for this paper.

\section{Conflict of interest}

JS has received lecture fees and has been a participant in meetings supported by Gilead, Pfizer, Merck-Sharp-Dohme (MSD), Teva, and Astellas. All other authors declare no conflicts of interest related to this study.

\section{Financial support}

None.

\section{Ethics}

The work described in this article has been carried out in accordance with The Code of Ethics of the World Medical Association (Declaration of Helsinki) for experiments involving humans; EU Directive 2010/63/ EU for animal experiments; Uniform Requirements for manuscripts submitted to biomedical journals. 


\section{References}

[1] Kontoyiannis DP, Marr KA, Park BJ, et al. Prospective surveillance for invasive fungal infections in hematopoietic stem cell transplant recipients, 2001-2006: overview of the Transplant-Associated Infection Surveillance Network (TRANSNET) Database. Clin Infect Dis 2010;50:1091-100.

[2] Lewis RE, Cahyame-Zuniga L, Leventakos K, et al. Epidemiology and sites of involvement of invasive fungal infections in patients with haematological malignancies: a 20-year autopsy study. Mycoses 2013;56:638-45.

[3] Lewis RE, Georgiadou SP, Sampsonas F, Chamilos G, Kontoyiannis DP. Risk factors for early mortality in haematological malignancy patients with pulmonary mucormycosis. Mycoses 2014;57:49-55.

[4] Vilela R, Mendoza L. Human pathogenic entomophthorales. Clin Microbiol Rev 2018;31:e00014-8.

[5] Roden MM, Zaoutis TE, Buchanan WL, et al. Epidemiology and outcome of zygomycosis: a review of 929 reported cases. Clin Infect Dis 2005;41:634-53.

[6] Manesh A, Rupali P, Sullivan MO, et al. Mucormycosis-A clinicoepidemiological review of cases over 10 years. Mycoses 2019;62:391-8.

[7] Muggeo P, Calore E, Decembrino N, et al. Invasive mucormycosis in children with cancer: A retrospective study from the Infection Working Group of Italian Pediatric Hematology Oncology Association. Mycoses 2019;62:165-70.

[8] Czyzewski K, Styczynski J, Giebel S, et al. Age-dependent determinants of infectious complications profile in children and adults after hematopoietic cell transplantation: lesson from the nationwide study. Ann Hematol 2019;98:2197-211.

[9] Czyzewski K, Galazka P, Fraczkiewicz J, et al. Epidemiology and outcome of invasive fungal disease in children after hematopoietic cell transplantation or treated for malignancy: Impact of national programme of antifungal prophylaxis. Mycoses 2019;62:990-8.

[10] Styczynski J. Infectious complications in children and adults with hematological malignancies. Acta Haematol Pol 2019;50:167-73.

[11] Donnelly JP, Chen SC, Kauffman CA, et al. Revision and Update of the Consensus Definitions of Invasive Fungal Disease From the European Organization for Research and Treatment of Cancer and the Mycoses Study Group Education and Research Consortium. Clin Infect Dis 2019 [Epub ahead of print].

[12] Styczynski J, Tridello G, Donnelly JP, et al. Protective environment for hematopoietic cell transplant (HSCT) recipients: The Infectious Diseases Working Party EBMT analysis of global recommendations on health-care facilities. Bone Marrow Transplant 2018;53:1131-8.
[13] Tomblyn M, Chiller T, Einsele H, et al. Guidelines for preventing infectious complications among hematopoietic cell transplantation recipients: a global perspective. Biol Blood Marrow Transplant 2009;15:1143-238.

[14] Skiada A, Lanternier F, Groll AH, et al. Diagnosis and treatment of mucormycosis in patients with hematological malignancies: guidelines from the 3rd European Conference on Infections in Leukemia (ECIL 3). Haematologica 2013;98:492-504.

[15] Leventakos K, Lewis RE, Kontoyiannis DP. Fungal infections in leukemia patients: how do we prevent and treat them? Clin Infect Dis 2010;50:405-15.

[16] Skiada A, Pagano L, Groll A, et al. Zygomycosis in Europe: analysis of 230 cases accrued by the registry of the European Confederation of Medical Mycology (ECMM) Working Group on Zygomycosis between 2005 and 2007. Clin Microbiol Infect 2011;17:1859-67.

[17] Xhaard A, Lanternier F, Porcher R, et al. Mucormycosis after allogeneic haematopoietic stem cell transplantation: a French Multicentre Cohort Study (2003-2008). Clin Microbiol Infect 2012;18:E396-400.

[18] Marty FM, Ostrosky-Zeichner L, Cornely OA, et al. Isavuconazole treatment for mucormycosis: a single-arm open-label trial and casecontrol analysis. Lancet Infect Dis 2016;16:828-37.

[19] Cornely OA, Alastruey-Izquierdo A, Arenz D, et al. Global guideline for the diagnosis and management of mucormycosis: an initiative of the European Confederation of Medical Mycology in cooperation with the Mycoses Study Group Education and Research Consortium. Lancet Infect Dis 2019;19:e405-21.

[20] Bays DJ, Thompson GR. Fungal infections of the stem cell transplant recipient and hematologic malignancy patients. Infect Dis Clin North Am 2019;33:545-66.

[21] Jeong W, Keighley C, Wolfe R, et al. Contemporary management and clinical outcomes of mucormycosis: A systematic review and metaanalysis of case reports. Int J Antimicrob Agents 2019;53:589-97.

[22] Jeong W, Keighley C, Wolfe R, et al. The epidemiology and clinical manifestations of mucormycosis: a systematic review and metaanalysis of case reports. Clin Microbiol Infect 2019;25:26-34.

[23] Kerezoudis P, Watts CR, Bydon M, et al. Diagnosis and treatment of isolated cerebral mucormycosis: patient-level data meta-analysis and Mayo Clinic experience. World Neurosurg 2019;123:425-34 e5.

[24] Vaughan C, Bartolo A, Vallabh N, Leong SC. A meta-analysis of survival factors in rhino-orbital-cerebral mucormycosis-has anything changed in the past 20 years? Clin Otolaryngol 2018;43:1454-64. 